\title{
Simultaneous control of magnetic topologies for reconfigurable vortex arrays
}

\author{
Mi-Young Im ${ }^{1,2}$, Peter Fischer ${ }^{3,4}$, Hee-Sung $\mathrm{Han}^{5}$, Andreas Vogel ${ }^{6}$, Min-Seung Jung ${ }^{2}$, Weilun Chao ${ }^{1}$, \\ Young-Sang $\mathrm{Yu}^{7}$, Guido Meier ${ }^{6,8,9}$, Jung-Il Hong ${ }^{2}$ and Ki-Suk Lee ${ }^{5}$
}

The topological spin textures in magnetic vortices in confined magnetic elements offer a platform for understanding the fundamental physics of nanoscale spin behavior and the potential of harnessing their unique spin structures for advanced magnetic technologies. For magnetic vortices to be practical, an effective reconfigurability of the two topologies of magnetic vortices, that is, the circularity and the polarity, is an essential prerequisite. The reconfiguration issue is highly relevant to the question of whether both circularity and polarity are reliably and efficiently controllable. In this work, we report the first direct observation of simultaneous control of both circularity and polarity by the sole application of an in-plane magnetic field to arrays of asymmetrically shaped permalloy disks. Our investigation demonstrates that a high degree of reliability for control of both topologies can be achieved by tailoring the geometry of the disk arrays. We also propose a new approach to control the vortex structures by manipulating the effect of the stray field on the dynamics of vortex creation. The current study is expected to facilitate complete and effective reconfiguration of magnetic vortex structures, thereby enhancing the prospects for technological applications of magnetic vortices.

NPG Asia Materials (2017) 9, e348; doi:10.1038/am.2016.199; published online 10 February 2017

\section{INTRODUCTION}

A magnetic vortex in a micron-sized ferromagnetic element consists of the central core (polarity, $p$ ) in which the magnetization is pointing into the out-of-plane direction either up $(p=+1)$ or down $(p=-1)$, and the in-plane magnetization (circularity, $c$ ) rotates around the central core in either the clockwise $(c=+1, \mathrm{CW})$ or counter-clockwise $(c=-1, \mathrm{CCW})$ direction. ${ }^{1,2}$ Because both circularity and polarity can be specified by two independent values, that is, $c= \pm 1$ and $p= \pm 1$, four distinct spin states can exist in a single magnetic element with the combination of circularity and polarity. Magnetic vortices have been intensively studied due to their compelling physical behavior ${ }^{3-7}$ and their potential in a wide range of applications such as data storage,, 89 signal transfer, ${ }^{10-12}$ logic devices, ${ }^{13}$ transistors ${ }^{14}$ and artificial skyrmion crystals. ${ }^{15-18}$ With respect to practical application of magnetic vortices in advanced nanotechnologies, one of the critical factors is the effective reconfigurability of two topologies, $c$ and $p$, particularly within large and densely packed arrays of magnetic elements. ${ }^{19,20}$ As a representative example, for successful achievement of vortex-based signal transfer and logic and transistor operations, the desired configurations of magnetic vortex states must be first established. ${ }^{10-14}$ Additionally, uniformly arranged vortex structures are also required to generate artificial skyrmion crystals based on magnetic vortices in proximity to perpendicularly magnetized thin films. ${ }^{15-18}$

For effective reconfiguration of magnetic vortex structures, one key issue is reliable and efficient control of both $c$ and $p$ in magnetic vortices, which is also vital for storage applications. Thus far, both static and dynamic studies on the control of vortex structures have been primarily dedicated to manipulation of either the $c$ or $p$ alone. ${ }^{8,21-31} \mathrm{~A}$ few attempts have been made to control both $c$ and $p$, but these attempts have been conducted without repetition, meaning that only a single event of control has been investigated. ${ }^{32,33}$ Therefore, reliability and repeatability for control of both topological features has not yet been addressed. In addition, multiple manipulation methods, for example, both in-plane and out-of-plane magnetic fields, have been applied to achieve control of both $c$ and p. Reliable and technically plausible control of both $c$ and $p$ with the application of a single control factor, such as either an

${ }^{1}$ Center for X-ray Optics, Lawrence Berkeley National Laboratory, Berkeley, CA, USA; ${ }^{2}$ Department of Emerging Materials Science, DGIST, Daegu, Korea; ${ }^{3}$ Materials Sciences Division, Lawrence Berkeley National Laboratory, Berkeley, CA, USA; ${ }^{4}$ Department of Physics, University of California, Santa Cruz, CA, USA; ${ }^{5}$ School of Materials Science and Engineering, KIST-UNIST Ulsan Center for Convergent Materials, Ulsan National Institute of Science and Technology (UNIST), UIsan, Korea; ${ }^{6}$ Institut für Angewandte Physik und Zentrum für Mikrostrukturforschung, Universität Hamburg, Hamburg, Germany; ${ }^{7}$ Advanced Light Source, Lawrence Berkeley National Laboratory, Berkeley, CA, USA; ${ }^{8}$ The Hamburg Centre for Ultrafast Imaging, Hamburg, Germany and ${ }^{9}$ Max-Planck Institute for the Structure and Dynamics of Matter, Hamburg, Germany Correspondence: Dr M-Y Im, Center for X-ray Optics, Lawrence Berkeley National Laboratory, 2-400, CXRO/LBNL, One Cyclotron Road, Berkeley, CA 94720, USA. E-mail: mim@lbl.gov

or Professor J-I Hong, Department of Emerging Materials Science, DGIST, 333 Technojungang-daero, Hyeonpung-myeon, Dalseong-gun, Daegu 42988, Korea.

E-mail: jihong@dgist.ac.kr

or Professor K-S Lee, 301-6, UNIST, 50, UNIST-gil, UIsan 44919, Korea.

E-mail: kisuk@unist.ac.kr

Received 15 April 2016; revised 26 October 2016; accepted 1 November 2016 
in-plane or out-of-plane field, and an in-depth understanding of relevant physics remain as challenges.

In the current investigation, we demonstrate that application of an in-plane magnetic field alone enables simultaneous control of $c$ and $p$ in an array of asymmetric permalloy $\left(\mathrm{Py}, \mathrm{Ni}_{80} \mathrm{Fe}_{20}\right)$ disks with one flat-edge side. The reliability in the control of vortex structures is found to be sensitive to the geometry of the nanodisk arrays, including the flat-edge ratio ( $r$, as defined in Figure 1$)$ and the disk height $(h)$. Based on a comprehensive understanding of the stray field effect on the dynamic process of vortex creation, we propose a new means of vortex-state control that enables the global and/or local reconfiguration of vortex structures in closely packed permalloy disks.

\section{MATERIALS AND METHODS}

\section{Sample preparation}

Py disk arrays were fabricated on X-ray transparent silicon-nitride membranes using e-beam lithography and sequential lift-off processes. The disk radius $(R)$ and interdisk distance $(d)$ were fixed at $R=500 \mathrm{~nm}$ and $d=200 \mathrm{~nm}$, and the disk height $(h)$ and flat-edge ratio $(r)$ were varied from $h=40$ to $100 \mathrm{~nm}$ and from $r=0.1 R$ to $0.2 R$.

\section{Imaging of magnetic vortex structures}

To observe the complete vortex structures (i.e., both circularity and polarity), we used magnetic full-field transmission soft X-ray microscopy (MTXM) at the Advanced Light Source (XM-1, beamline 6.1.2.). ${ }^{34}$ In-plane and out-of-plane magnetizations were respectively imaged by mounting the specimens at $60^{\circ}$ and $90^{\circ}$ angles with respect to the incident X-ray beam direction. The magnetic contrast at XM-1 is created by an X-ray magnetic circular dichroism mechanism. The X-ray beam energy was set at the $\mathrm{Fe}_{3}$ edge $(707 \mathrm{eV})$ of the X-ray absorption spectra. To enhance the magnetic contrast and eliminate any non-magnetic background, the images collected in the remanent state were normalized by a reference image recorded at the saturation state. To saturate the Py disks, an in-plane magnetic field of +100 or $-100 \mathrm{mT}$ was applied parallel to the flat edge of disks, as shown in Figure 1.

\section{Micromagnetic simulations}

Micromagnetic simulations were conducted with disks of $2 R=999 \mathrm{~nm}$, $h=40 \mathrm{~nm}, r=99 \mathrm{~nm}(\sim 0.2 R)$ and an interdisk distance of $d=201 \mathrm{~nm}$, the dimensions of which are nearly identical to those of the disks $(2 R=1000 \mathrm{~nm}$, $h=40 \mathrm{~nm}, r=100 \mathrm{~nm}(\sim 0.2 R)$ and $d=200 \mathrm{~nm})$ studied in the experiments. The lateral dimensions of the disks and the interdisk distance were multiples of $3 \mathrm{~nm}$ due to the cell size of $3 \times 3 \times 5 \mathrm{~nm}^{3}$ chosen for the simulations. The micromagnetic simulations were performed using the object-oriented micromagnetic framework (OOMMF) code, ${ }^{35}$ which numerically solves the Landau-Lifshitz-Gilbert equation ${ }^{36,37}$ for the local magnetization vector $\mathbf{M}(\mathbf{r}, t): \partial \mathbf{M} / \partial t=-\gamma\left(\mathbf{M} \times \mathbf{H}_{\text {eff }}\right)+(\alpha /|\mathbf{M}|)(\mathbf{M} \times \partial \mathbf{M} / \partial t)$ with phenomenological damping constant $\boldsymbol{\alpha}$, gyromagnetic ratio $\gamma$ and effective field $\mathrm{H}_{\text {eff }}$.

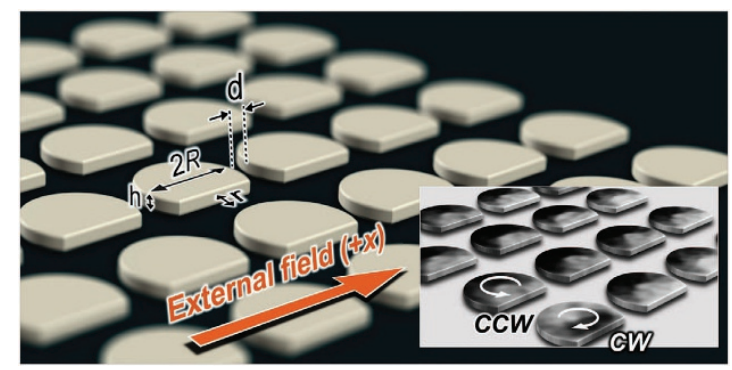

Figure 1 Magnetic imaging of asymmetrically shaped disks. Schematic diagram of asymmetric disks with radius $R=500 \mathrm{~nm}$, height $h=100 \mathrm{~nm}$ and edge ratio $r=0.2 R$ within an array with an interdisk distance of $d=200 \mathrm{~nm}$. Insets show representations of MTXM images of the in-plane magnetic components observed in a positive field sequence from +100 to $0 \mathrm{mT}$.
The standard material parameters for Py were used: saturation magnetization $M_{\mathrm{s}}=800 \mathrm{kA} \mathrm{m}^{-1}$; exchange stiffness $A_{\mathrm{ex}}=13 \mathrm{pJ} \mathrm{m}^{-1}$; damping constant $\alpha=0.01$; and zero magnetocrystalline anisotropy. Periodic boundary conditions were used in the simulations to take account of stray fields induced by adjacent disks within the arrays of disks.

\section{RESULTS AND DISCUSSION}

Direct observation of magnetic vortex structures

Figure 1 shows the schematic diagram of a Py disk array with a radius of $R=500 \mathrm{~nm}$, a height of $h=100 \mathrm{~nm}$, an interdisk distance of $d=200 \mathrm{~nm}$ and a flat-edge ratio of $r=0.2 R$. The creation of vortex structures in the disk array was achieved using a positive field sequence: a magnetic field of $+100 \mathrm{mT}$ is applied in the positive $x$ direction $\left(H=+H_{x}\right)$ parallel to the flat edges of disk elements to fully saturate the disks and is subsequently relaxed to the remanent state at $0 \mathrm{mT}$ by turning off the magnetic field. The inset in Figure 1 illustrates MTXM images of the in-plane spin configurations observed in the array after the positive field sequence. The direction of magnetization in the disk plane is indicated with a white arrow. The in-plane magnetic contrast allows the value of $c$ to be determined. It is clearly evident that with the positive field sequence, the preferably created circularity is $\operatorname{CCW}(c=-1)$. To determine whether any field-direction effect exists on the type of created circularity, we also observed the vortex structures formed after the following sequence: a field of $-100 \mathrm{mT}$ is applied in the negative $x$ direction $\left(H=-H_{x}\right)$ and is subsequently turned off to $0 \mathrm{mT}$ (hereafter referred to as the negative field sequence). In Figure $2 \mathrm{a}$, we show a representative series of images for the in-plane domain structures taken from two different field sequences in the disk arrays with edge ratios $r=0.1 R$ and $r=0.2 R$. In both arrays, the CW $(c=+1)$ circularity is created by the negative field sequence, and the CCW $(c=-1)$ circularity is created by the positive field sequence. This result shows that circularity can be selectively created in the asymmetric disks by altering the direction of the in-plane field. It should be noted that the rotational sense of circularity selected by each field sequence in the disks within the array shown in Figure $2 \mathrm{a}$ is opposite to the one formed in an isolated disk with the identical field sequence. ${ }^{24,26,27}$ Under the positive (negative) field sequence, CCW (CW) circularity is generated in the disks within the array, whereas CW (CCW) circularity is created in an isolated disk. ${ }^{24,26,27}$ To understand the change in the type of created circularity between disks within the array and a single isolated disk, we considered the effect of the stray field on the vortex creation process. Based on micromagnetic simulations for vortex formation with and without consideration of the stray field, we confirmed that the dynamics of vortex creation could be significantly altered by the stray field. Under the influence of the stray field, the dynamic process of vortex formation in the positive (negative) field sequence proceeds toward creation of the CCW (CW) circularity, as observed in disks within the array (Figure 2a). The detailed dynamic procedure and the stray field effect on the dynamics are discussed later.

\section{Controllability of circularity in asymmetric disk arrays}

The reliability of circularity control (controllability) was quantitatively investigated by switching the direction of the external field for various arrays of disks with different disk heights and flat-edge ratios, as shown in Figure 2 b. To accumulate statistically meaningful data, the experimental procedure schematized in Figure 2a was repeated 40 times, and vortex structures in 25 individual disks were examined in each array. The generation rate of an identical circularity in any given disk within 40 repetitions was counted as the metric for controllability, where the controllability is defined as $100 \%(0 \%)$ 
if the generation rate of CCW circularity is $100 \%$ (50\%) within 40 repeated cycles with the positive field sequence (for which CCW circularity is predominantly generated). In Figure $2 \mathrm{~b}$, the error bar represents the standard deviation of controllability over the 25 disks. The reliability for formation of a vortex structure with the same circularity in asymmetric disks is certainly improved compared with the case of symmetric circular disks. The reliability of circularity control is greater than $95 \%$ in asymmetric disks with $h=100 \mathrm{~nm}$, as shown in Figure 2b, whereas the probability for formation of vortex structures with the same circularity determined from statistical
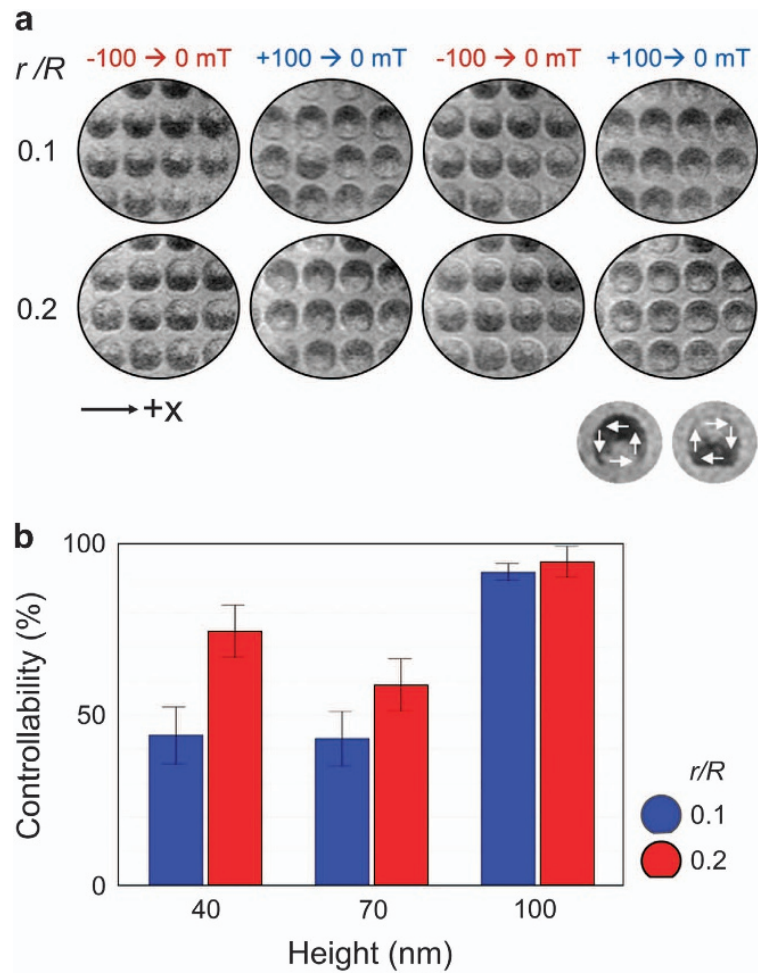

Figure 2 Controllability of circularity in asymmetric disk arrays with different geometries. (a) MTXM images of in-plane domain structures taken from four successive measurements by alternating positive and negative field sequences in the disk arrays with $R=500 \mathrm{~nm}, h=100 \mathrm{~nm}$ and $d=200 \mathrm{~nm}$. (b) Controllability of circularity by altering the direction of the applied field investigated in disk arrays $(R=500 \mathrm{~nm}, d=200 \mathrm{~nm})$ with different edge ratios and disk heights. The values are obtained from statistical analysis of generation probabilities of $c$ in 1000 vortex formation events for each array. The error bar represents the standard deviation of controllability in 25 individual disks. measurements (20 repetitions, 25 individual disks) is less than $35 \%$ in the case of symmetric full disks with the same height of $100 \mathrm{~nm}$. In Figure $2 \mathrm{~b}$, we find that the controllability is sensitive to the geometry of the disk arrays. As the flat-edge ratio increases from $r=0.1 R$ to $0.2 R$, the controllability increases, and disks of $h=100 \mathrm{~nm}$ show higher controllability than those of $h=40$ or $70 \mathrm{~nm}$. The high controllability of greater than $90 \%$ observed in the 100 -nm-thick disks, regardless of the edge ratio, might be due to the less pronounced impact of thermal fluctuations associated with the large volume of disks. Indeed, thermal fluctuation ${ }^{38}$ is an inherent factor that hinders reliable control of magnetic processes. ${ }^{39,40}$ The intensity of thermal fluctuation occurs in inverse proportion to an activation volume, which is related to the thermal effect. ${ }^{41}$ In vortex formation, the disk volume could be considered as an activation volume $e^{42}$ because the formation process undergoes multiple steps of nucleation and annihilation of the vortex and anti-vortex occurring in the vortex core and also over the entire disk volume. ${ }^{43}$ The effective thermal effect is reduced by a factor of 2.5 as the disk height increases from 40 to $100 \mathrm{~nm}$, that is, disks of $h=100 \mathrm{~nm}$ are less prone to thermal fluctuations than the thinner disks. Consequently, in larger-volume disks, the same type of vortex structure can be more reliably created.

\section{Simultaneous control of circularity and polarity in asymmetric disks}

For disks of $h=100 \mathrm{~nm}$ and $r=0.2 R$, which exhibit approximately $97 \%$ accuracy in circularity control, we verified the simultaneous control of $c$ and $p$ through direct imaging of circularity and polarity. Figure 3a shows MTXM images of in-plane and out-of-plane magnetic structures observed in an identical region of the array. In the out-of-plane MTXM images, the white and black spots in the centers of the disks indicate down and up polarities, respectively. Remarkably, the change of field direction triggers switching of the circularity and the polarity. CW circularity $(c=+1)$ with a down polarity $(p=-1)$ is formed after the positive field sequence, whereas CCW circularity $(c=-1)$ with an up polarity $(p=+1)$ is most often generated after the negative field sequence. A simplified illustration of this observed phenomenon is shown in Figure $3 \mathrm{~b}$. The results indicate that simultaneous control of $c$ and $p$ is feasible using a single manipulation process, that is, application of an in-plane magnetic field in the asymmetric disks. This result suggests the possibility of highly efficient reconfiguration of the magnetic-vortex structures in nanodisk arrays. Our result is unexpected and intriguing because only $c$ has been considered controllable in asymmetric disks. ${ }^{24-28}$ Simultaneous control of $c$ and $p$ is thought to be related to the asymmetric nature in the formation process of vortex states, as reported in our previous work. ${ }^{7}$ It was found that two different vortex state groups with $c p=+1$ and a

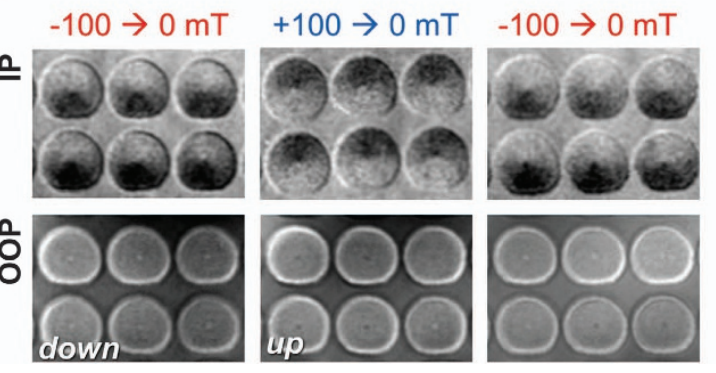

b

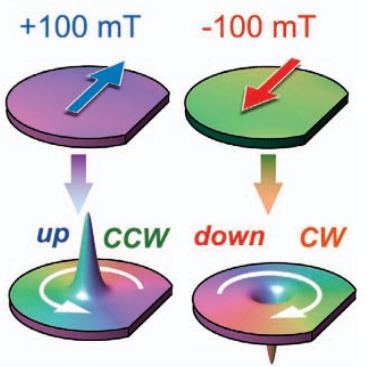

Figure 3 Simultaneous control of circularity and polarity in asymmetric disks. (a) MTXM images of in-plane and out-of-plane magnetic structures observed in an identical region of the disk array $(R=500 \mathrm{~nm}, h=100 \mathrm{~nm}$ and $r=0.2 R)$. (b) Simplified illustration of control of complete vortex structure, that is, both $c$ and $p$, by changing the direction of the in-plane field. 
$c p=-1$ are not energetically equivalent in a single circular disk due to both intrinsic and extrinsic factors. Either $c p=+1$ or $c p=-1$ is more stable than the other, and the energetically favored vortex group predominantly forms. In the case of asymmetric disks (Figure 3a), $c p=-1$ is more favored than $c p=+1$. If $c=+1$ switches to $c=-1$, $p$ tends to flip from $p=-1$ to $p=+1$ to form $c p=-1$. Notably, $c$ and $p$ are not independent, but they are linked with each other in that $c$ and $p$ tend to switch together. This intrinsic connection between $c$ and $p$ could be a critical feature that must be seriously considered for individual control of $c$ and $p$. In contrast to circular disks, circularity in asymmetric disks is controllable by altering the direction of the magnetic field, which allows for simultaneous control of $c$ and $p$ in such disks. The reliability of simultaneous control of $c$ and $p$, as determined by a statistical ensemble of 300 events (15 repetitions, 20 individual disks), was found to be $77 \pm 17$ and $79 \pm 16 \%$ in disks of $100 \mathrm{~nm}$ thickness with $0.2 R$ and $0.1 R$ edge ratios, respectively. The errors correspond to the s.d. of reliability for the 20 disks. It
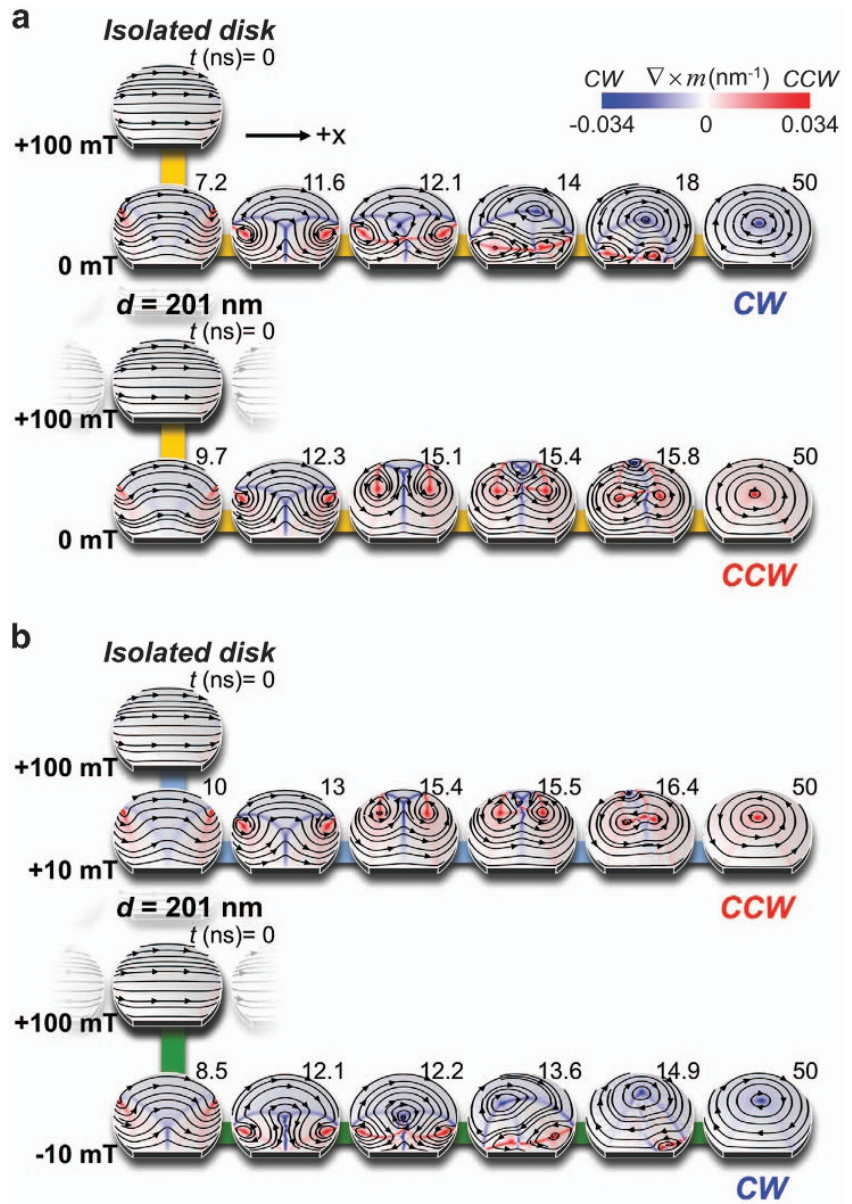

Figure 4 Control of vortex structures by manipulation of the stray field effect on the dynamic process of vortex creation. (a) Series of snapshot images taken from simulations of vortex formation in an isolated disk (upper) and in a disk within a $d=201 \mathrm{~nm}$ array (lower). The disks have dimensions of $2 R=999 \mathrm{~nm}$ radius, $h=40 \mathrm{~nm}$ height and $r=99 \mathrm{~nm}$ flat-edge ratio $(\sim 0.2 R)$. In this work, the disks were immediately relaxed to $0 \mathrm{mT}$ from a $100 \mathrm{mT}$ saturation state without application of a control field. (b) Dynamic process observed by simulations with control fields of $+10 \mathrm{mT}$ and $-10 \mathrm{mT}$ additionally applied during the relaxation process of an isolated disk (upper) and a disk within the array (lower), respectively. The color on the disk surface corresponds to the curl of in-plane magnetization. The blue and red colors indicate the CW and CCW circularities, respectively. should be noted that the data obtained from the statistical ensemble cover any possible randomness or imperfection of vortex control that can exist in certain of the disks, as shown in one of the six disks in the lower-middle panel and two of the six disks in the lower-right panel, as indicated by white dots in Figure 3a. The reliability of vortex control can differ from one disk to another, mostly due to randomly generated surface-related extrinsic factors such as roughness and defects. It is expected that the accuracy of controlling both $c$ and $p$ can be further enhanced by optimizing the geometries of asymmetric disk arrays.

New approach for control of vortex structures in asymmetric disks Finally, we propose a new approach to controlling $c$ in asymmetrically shaped disks. As demonstrated in Figure 3, because simultaneous control of $c$ and $p$ is feasible when $c$ is reliably controlled, this method could replace that involving alteration of the direction of an in-plane field for control of both $c$ and $p$ in asymmetric disks. As noted in Figure 2, the stray field is the critical magnetic factor for determination of finally formed $c$ states in asymmetric disks because it significantly affects the dynamics in the early stage of the vortex formation process. ${ }^{43}$ A certain type of circularity, ether $c=+1$ or $c=-1$, is selected according to whether the stray field exists or not. Our approach to the control of $c$ is to manipulate the stray field effect on the dynamic process of vortex creation using an additional, static and uniform field (hereafter, the control field). The control field can create an effective stray field environment of closely packed disks that resembles one of an isolated disk and vice versa.

First, to fully understand the influence of the stray field on the process of vortex structure formation, micromagnetic simulations of the dynamics of vortex formation in an isolated element and the same element with neighboring identical elements have been performed without considering the control field. The two disks were set to the same geometry of $2 R=999 \mathrm{~nm}, h=40 \mathrm{~nm}, r=99 \mathrm{~nm}(\sim 0.2 R)$ and an interdisk distance of $d=201 \mathrm{~nm}$, parameters that are nearly equal to those $(2 R=1000 \mathrm{~nm}, h=40 \mathrm{~nm}, r=100 \mathrm{~nm}(\sim 0.2 R)$ and $d=200 \mathrm{~nm})$ of the disk studied in the experiments. Figure 4 a shows images of the simulated dynamic processes in an isolated single disk (upper) and in a disk within the array of $d=201 \mathrm{~nm}$ (lower). The color on the disk surface represents the curl of in-plane magnetization $(m)$. The red (blue) color indicates the CCW (CW) winding direction of $m$, and the arrow indicates the direction of in-plane magnetization on the disk surface. To trigger vortex creation, the disks were initially saturated at $+100 \mathrm{mT}$ and subsequently relaxed to $0 \mathrm{mT}$ within $\tau=4.4 \mathrm{~ns}$. The strength of the stray field generated in the disk array of $d=201 \mathrm{~nm}$ during the process was estimated to be approximately $10 \mathrm{mT}$ in the $+x$ direction. In the early stage of vortex formation $(\leqslant 7.2 \mathrm{~ns}$ for isolated disks (upper), $\leqslant 9.7 \mathrm{~ns}$ for a disk within an array (lower)), nearly identical dynamic processes were observed in the two disks. Two vortices are injected from the left and right sides of the disk, respectively, and they move closer to each other. Because these disks have the same CCW circularity (red), a cusp-shaped magnetization configuration is formed between them and is transformed into an oxbow-shaped configuration ( $t=11.6 \mathrm{~ns}$ (upper) and $12.3 \mathrm{~ns}$ (lower)). As the dynamics progress, an additional CW vortex appears $(t=12.1 \mathrm{~ns}$ (upper) and $15.4 \mathrm{~ns}$ (lower)). The effect of the stray field becomes obvious from comparison of the isolated disk at $t=14 \mathrm{~ns}$ (upper) with the disk within the array at $15.8 \mathrm{~ns}$ (lower). In the isolated disk, the initially injected CCW vortices move toward the flat edge of the disk, and the newly created CW vortex (blue) is located in the central region. However, due to the stray field in the disk within the array of $d=201 \mathrm{~nm}$, the two CCW vortices remain at the center, and the $\mathrm{CW}$ vortex is annihilated at the round edge. Consequently, in 
a

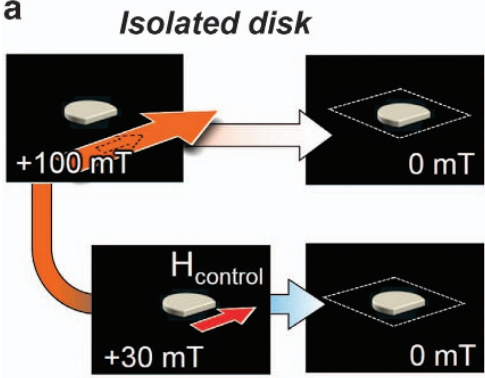

b

$d=201 \mathrm{~nm}$

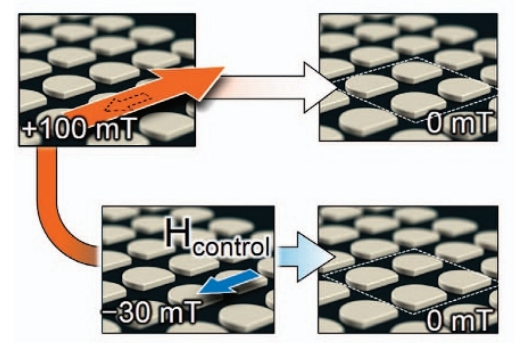

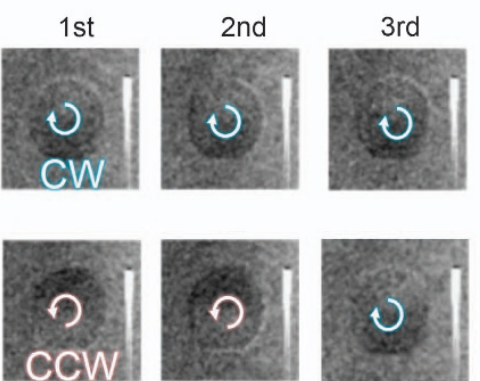

1 st
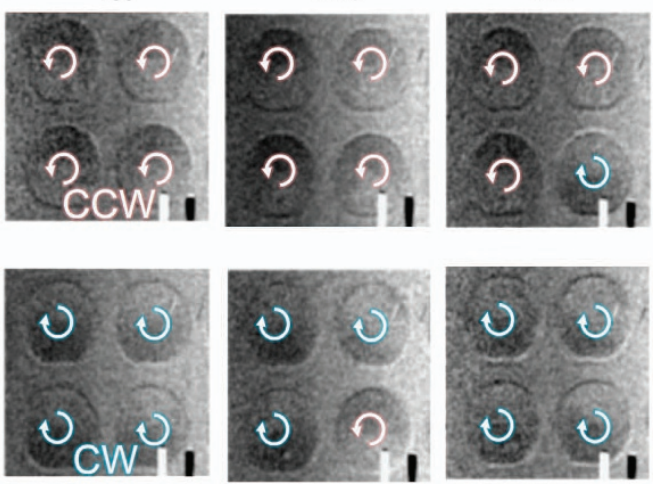

Figure 5 MTXM images of finally formed circularities in the positive field sequence $(+100 \mathrm{mT}$ to $0 \mathrm{mT})$ without and with the control field of $H_{\text {control }}=+30 \mathrm{mT}$ and $-30 \mathrm{mT}$ applied during vortex formation in an isolated disk (a) and in disks within an array of $d=201 \mathrm{~nm}$ (b), respectively. The dimensions of the disks are $2 R=1 \mu \mathrm{m}, h=40 \mathrm{~nm}$ and $r=100 \mathrm{~nm}(\sim 0.2 R)$, which are nearly equal to those $(2 R=999 \mathrm{~nm}, h=40 \mathrm{~nm}$ and $r=99 \mathrm{~nm}(\sim 0.2 R))$ of the disk in the simulations.

the isolated disk, the inner-disk CW vortex becomes the final vortex structure, whereas in the disk within the array, one of the initially injected CCW vortices survives as the ultimate vortex. In summary, the stray field in the $d=201 \mathrm{~nm}(\sim 10 \mathrm{mT})$ array stimulates the creation of CCW circularity in the positive field sequence from +100 to $0 \mathrm{mT}$.

To clarify our concept of circularity control using a control field, we again conducted micromagnetic simulations in identical systems. The disks were relaxed to $0 \mathrm{mT}$ from the fully saturated state at $+100 \mathrm{mT}$, according to the procedure outlined in Figure 4a. However, in this simulation, control fields of $\pm 10 \mathrm{mT}$ were additionally applied at the moment when the $100 \mathrm{mT}$ saturation field was turned off. By turning on the control field of $+10 \mathrm{mT}(-10 \mathrm{mT})$ in the positive field sequence, the saturation field of $+100 \mathrm{mT}$ is reduced directly to $+10 \mathrm{mT}(-10 \mathrm{mT})$. The purpose of the control field is to create an environment similar to that in which the stray field exists in an isolated disk and to create the condition under which no stray field exists in the disk within the array by countering the influence of the stray field induced by adjacent disks within the array. The control-field strength $(10 \mathrm{mT})$ was chosen based on the results shown in Figure 4a, in which case the stray field strength in the disk array of $d=201 \mathrm{~nm}$ was approximately $10 \mathrm{mT}$. The direction of the control field was set as $+x$ for the isolated disk and $-x$ for the disk within the array, considering the fact that the stray field in the array of $d=201 \mathrm{~nm}$ occurred in the $+x$ direction (Figure $4 a$ ). Snapshot images taken from the simulations are displayed in Figure $4 \mathrm{~b}$. The dynamics in the isolated disk with the applied $+10 \mathrm{mT}$ control field (upper) are analogous to those observed in the disk within the array (Figure 4a). The initially injected CCW vortices (red) shift to the center of the disk during the annihilation of the CW vortex (blue) at the $16.4 \mathrm{~ns}$ stage, and consequently, the final vortex is CCW, as in the case of the disk within the $d=201 \mathrm{~nm}$ array with no control field (Figure 4a). At the same time, the dynamic process in the disk within the array with the induced $-10 \mathrm{mT}$ control field (lower) was almost identical to that observed in the isolated disk (Figure 4a). The CW vortex newly created at the $12.2 \mathrm{~ns}$ stage is located in the middle of the disk with the two CCW circularities pushed toward the edge (13.6 ns), and the CW vortex finally forms. The simulation results demonstrate that the effect of the stray field on the dynamic process of vortex creation can be manipulated by the applied control field and that the finally created vortex structure is consequently controlled.

This proposed approach was also confirmed experimentally. Figure 5 shows a series of images for in-plane domain structures observed in an isolated disk (a) and in disks within an array of $d=201 \mathrm{~nm}$ after their relaxation from the saturation state to the remanent state without and with application of the control field of $+30 \mathrm{mT}$ and $-30 \mathrm{mT}$, respectively. The positive $H_{\text {control was applied to }}$ an isolated disk to mimic the stray field existing in the arrays, and the negative $H_{\text {control }}$ was applied to the disks within the array to compensate the effect of the stray field induced by the adjacent disks. We applied $H_{\text {control }}$ with the same external field magnet used in saturation of the disks. To reproduce the procedures performed in the simulations, $H_{\text {control }}$ was applied immediately after the saturation field of $+100 \mathrm{mT}$ was turned off, and the finally created vortex structures were observed after turning off $H_{\text {control }}$. Within three repeated measurements, as shown in Figure 5, by applying $H_{\text {control }}$ during the formation process of vortex structure, the created circularity switches from CW $(c=1)$ to CCW $(c=-1)$ in the isolated disk and CCW $(c=-1)$ to $\mathrm{CW}(c=1)$ in disks within the array, although a few exceptions could also be observed occasionally, in good agreement 
with the simulation results. In the experiment, $H_{\text {control }}$ stronger than $10 \mathrm{mT}$ was preferred to reliably manipulate the stray field effect. It is thought that the stray field from neighboring disks in the array might be not exactly $10 \mathrm{mT}$ in all cases but exceeds $10 \mathrm{mT}$ in real samples with non-ideal geometries. We also found that the reliability for control of the vortex structure using $H_{\text {control }}$ is improved as the strength of the control field increases. Based on 20 repeated measurements, it was verified that reliability for the control of circularity can exceed $80 \%$ with $H_{\text {control }}= \pm 50 \mathrm{mT}$. In this manner, for both an isolated disk and a disk within an array, the circularity can be simply controlled by turning the additional $H_{\text {control }}$ on or off, without any need to alter any aspects of array geometry such as the interdisk distance. Moreover, this method offers the possibility of selective control of specific vortex structures in closely packed disks with a simple electrode architecture, thereby also enabling local reconfiguration of magnetic vortices in the disk arrays. By applying current through an electrode during the relaxation of the disks from the saturation state to the remanent state, circularity is controllable using the Oersted field thus generated. The Oersted field induces a controlfield effect equal to that shown in Figures $4 \mathrm{~b}$ and 5, and the latter field can control the stray field from the adjacent disks within the arrays. Any type of electrode is applicable, although the field generated must be sufficiently strong to offset the effect of the stray field that already exists within the arrays.

\section{CONCLUSION}

We report that simultaneous control of vortex circularity and polarity can be achieved using a simple procedure that applies an in-plane magnetic field, and the reliability of this approach can be enhanced with optimized geometry of the asymmetric disk arrays. Because reliable and simultaneous control of $c$ and $p$ could be crucially beneficial to effective reconfiguration of magnetic vortex structures within arrays, this work advances the potential for applications of magnetic vortices in advanced magnetic nanotechnologies.

\section{CONFLICT OF INTEREST}

The authors declare no conflict of interest.

\section{ACKNOWLEDGEMENTS}

This work was supported by the National Research Foundation (NRF) of Korea funded by the Ministry of Education, Science and Technology (MEST) (2012K1A4A3053565, 2014R1A2A2A01003709, 2015M3D1A1070465 and 2016K1A3A7A09005336), by the KIST-UNIST partnership program (1.160097.01/2V05150). Work at the ALS was supported by the Director, Office of Science, Office of Basic Energy Sciences, Scientific User Facilities Division of the U.S. Department of Energy under Contract No. DE-AC02-05CH11231. PF acknowledges support by the U.S. Department of Energy, Office of Science, Office of Basic Energy Sciences, Materials Sciences and Engineering Division under Contract No. DE-AC02-05CH11231 within the Non-Equilibrium Magnetic Materials Program (MSMAG). AV and GM acknowledge financial support from the Deutsche Forschungsgemeinschaft via SFB 668 'Magnetism from the Single Atom to the Nanostructure', via Graduiertenkolleg 1286 'Functional Metal-Semiconductor Hybrid Systems', and via excellence cluster 'The Hamburg Centre for Ultrafast Imaging—Structure, Dynamics and Control of Matter on the Atomic Scale'.

1 Shinjo, T., Okuno, T., Hassdorf, R., Shigeto, K. \& Ono, T. Magnetic vortex core observation in circular dots of permalloy. Science 289, 930-932 (2000).
2 Wachowiak, A., Wiebe, J., Bode, M., Pietzsch, O., Morgenstern, M. \& Wiesendanger, R. Direct observation of internal spin structure of magnetic vortex cores. Science 298, 577-580 (2002).

3 Tanigaki, T., Takahashi, Y., Shimakura, T., Akashi, T., Tsuneta, R., Sugawara, A. \& Shindo, D. Three-dimensional observation of magnetic vortex cores in stacked ferromagnetic discs. Nano Lett. 15, 1309-1314 (2015).

4 Fischer, P., Im, M.-Y., Kasai, S., Yamada, K., Ono, T. \& Thiaville, A. X-ray imaging of vortex cores in confined magnetic structures. Phys. Rev. B 83, 212402 (2011).

5 Adolff, C. F., Hänze, M., Pues, M., Weigand, M. \& Meier, G. Gyrational modes of benzenelike magnetic vortex molecules. Phys. Rev. B 92, 024426 (2015).

6 Behncke, C., Hänze, M., Adolff, C. F., Weigand, M. \& Meier, G. Band structure engineering of two-dimensional magnonic vortex crystals. Phys. Rev. B 91, 224417 (2015).

7 Im, M.-Y., Fischer, P., Yamada, K., Sato, T., Kasai, S., Nakatani, Y. \& Ono, T. Symmetry breaking in the formation of magnetic vortex states in a permalloy nanodisk. Nat. Commun. 3, 983 (2012).

8 Van Waeyenberge, B., Puzic, A., Stoll, H., Chou, K. W., Tyliszczak, T., Hertel, R., Fahnle, M., Bruckl, H., Rott, K., Reiss, G., Neudecker, I., Weiss, D., Back, C. H. \& Schutz, G. Magnetic vortex core reversal by excitation with short bursts of an alternating field. Nature 444, 461-464 (2006).

9 Mitin, D., Nissen, D., Schädlich, P., Arekapudi, S. S. P. K. \& Albrecht, M. Single vortex core recording in a magnetic vortex lattice. J. Appl. Phys. 115, 063906 (2014).

10 Han, D. S., Vogel, A., Jung, H., Lee, K. S., Weigand, M., Stoll, H., Schutz, G., Fischer, P., Meier, G. \& Kim, S. K. Wave modes of collective vortex gyration in dipolarcoupled-dot-array magnonic crystals. Sci. Rep. 3, 2262 (2013).

11 Sugimoto, S., Fukuma, Y., Kasai, S., Kimura, T., Barman, A. \& Otani, Y. Dynamics of coupled vortices in a pair of ferromagnetic disks. Phys. Rev. Lett. 106, 197203 (2011).

12 Vogel, A., Kamionka, T., Martens, M., Drews, A., Chou, K. W., Tyliszczak, T., Stoll, H., Van Waeyenberge, B. \& Meier, G. Coupled vortex oscillations in spatially separated permalloy squares. Phys. Rev. Lett. 106, 137201 (2011).

13 Jung, H., Choi, Y.-S., Lee, K.-S., Han, D.-S., Yu, Y.-S., Im, M.-Y., Fischer, P. \& Kim, S.-K. Logic operations based on magnetic-vortex-state networks. ACS Nano 6, 3712-3717 (2012).

14 Kumar, D., Barman, S. \& Barman, A. Magnetic vortex based transistor operations. Sci. Rep. 4, 4108 (2014)

15 Sun, L., Cao, R. X., Miao, B. F., Feng, Z., You, B., Wu, D., Zhang, W., Hu, A. \& Ding, H. F. Creating an artificial two-dimensional Skyrmion crystal by nanopatterning. Phys. Rev. Lett. 110, 167201 (2013).

16 Gilbert, D. A., Maranville, B. B., Balk, A. L., Kirby, B. J., Fischer, P., Pierce, D. T., Unguris, J., Borchers, J. A. \& Liu, K. Realization of ground-state artificial skyrmion lattices at room temperature. Nat. Commun. 6, 8462 (2015).

17 Miao, B. F., Sun, L., Wu, Y. W., Tao, X. D., Xiong, X., Wen, Y., Cao, R. X., Wang, P., Wu, D., Zhan, Q. F., You, B., Du, J., Li, R. W. \& Ding, H. F. Experimental realization of two-dimensional artificial skyrmion crystals at room temperature. Phys. Rev. B 90, 174411 (2014).

18 Li, J., Tan, A., Moon, K. W., Doran, A., Marcus, M. A., Young, A. T., Arenholz, E., Ma, S., Yang, R. F., Hwang, C. \& Qiu, Z. Q. Tailoring the topology of an artificial magnetic skyrmion. Nat. Commun. 5, 4704 (2014).

19 Streubel, R., Kronast, F., Rößler, U. K., Schmidt, O. G. \& Makarov, D. Reconfigurable large-area magnetic vortex circulation patterns. Phys. Rev. B 92, 104431 (2015).

20 Martín, J. I., Vélez, M., Hoffmann, A., Schuller, I. K. \& Vicent, J. L. Artificially induced reconfiguration of the vortex lattice by arrays of magnetic dots. Phys. Rev. Lett. 83, 1022 (1999).

21 Hertel, R., Gliga, S., Fahnle, M. \& Schneider, C. M. Ultrafast nanomagnetic toggle switching of vortex cores. Phys. Rev. Lett. 98, 117201 (2007).

22 Yu, Y.-S., Lee, K.-S., Jung, H., Choi, Y.-S., Yoo, M.-W., Han, D.-S., Im, M.-Y., Fischer, P. \& Kim, S.-K. Polarization-selective vortex-core switching by tailored orthogonal Gaussian-pulse currents. Phys. Rev. B 83, 174429 (2011).

23 Bohlens, S., Krger, B., Drews, A., Bolte, M., Meier, G. \& Pfannkuche, D. Current controlled random-access memory based on magnetic vortex handedness. Appl. Phys. Lett. 93, 142508 (2008)

24 Kimura, T., Otani, Y., Masaki, H., Ishida, T., Antos, R. \& Shibata, J. Vortex motion in chilarity-controlled pair of magnetic disks. Appl. Phys. Lett. 90, 132501 (2007).

25 Huang, L., Schofield, M. A. \& Zhu, Y. Control of double-vortex domain configurations in a shape-engineered trilayer nanomagnet system. Adv. Mater. 22, 492-495 (2010).

26 Wu, K.-M., Horng, L., Wang, J.-F., Wu, J.-C., Wu, Y.-H. \& Lee, C.-M. Influence of asymmetry on vortex nucleation and annihilation in submicroscaled permalloy disk array. Appl. Phys. Lett. 92, 262507 (2008).

27 Schneider, M., Hoffmann, H. \& Zweck, J. Magnetic switching of single vortex permalloy elements. Appl. Phys. Lett. 79, 3113 (2001).

28 Dumas, R. K., Gilbert, D. A., Eibagi, N. \& Liu, K. Chirality control via double vortices in asymmetric co dots. Phys. Rev. B 83, 060415 (2011).

29 Giesen, F., Podbielski, J., Botters, B. \& Grundler, D. Vortex circulation control in large arrays of asymmetric magnetic rings. Phys. Rev. B 75, 184428 (2007).

30 Haldar, A. \& Adeyeye, A. O. Vortex chirality control in circular disks using dipole-coupled nanomagnets. Appl. Phys. Lett. 106, 032404 (2015).

31 Uhlir, V., Urbanek, M., Hladik, L., Spousta, J., Im, M. Y., Fischer, P., Eibagi, N., Kan, J. J., Fullerton, E. E. \& Sikola, T. Dynamic switching of the spin circulation in tapered magnetic nanodisks. Nat. Nanotechnol. 8, 341-346 (2013).

32 Jaafar, M., Yanes, R., Perez de Lara, D., Chubykalo-Fesenko, O., Asenjo, A., Gonzalez, E. M., Anguita, J. V., Vazquez, M. \& Vicent, J. L. Control of the chirality and polarity of magnetic vortices in triangular nanodots. Phys. Rev. B 81, 054439 (2010). 
33 Shimon, G., Ravichandar, V., Adeyeye, A. O. \& Ross, C. A. Simultaneous control of vortex polarity and chirality in thickness-modulated [Co/Pd]n/Ti/Ni80Fe20 disks. Appl. Phys. Lett. 105, 152408 (2014).

34 Fischer, P., Kim, D.-H., Chao, W., Liddle, J. A., Anderson, E. H. \& Attwood, D. T. Soft X-ray microscopy of nanomagnetism. Mater. Today 9, 26-33 (2006).

35 Donahue, M. J. \& Porter, D. G. OOMMF User's Guide Version 1.0 Interagency Report NISTIR 6376, National Institute of Standards and Technology: Gaitherburg, MD, (1999).

36 Landau, L. D. \& Lifshitz, E. M. Theory of the dispersion of magnetic permeability in ferromagnetic bodies. Phys. Z. Sowjetunion 8, 153 (1935).

37 Gilbert, T. L. A phenomenological theory of damping in ferromagnetic materials. IEEE Trans. Magn. 40, 3443-3449 (2004).

38 Brown, W. F. Thermal fluctuations of a single-domain particle. Phys. Rev. 130, 1677-1686 (1963).

39 Shpyrko, O. G., Isaacs, E. D., Logan, J. M., Feng, Y., Aeppli, G., Jaramillo, R., Kim, H. C., Rosenbaum, T. F., Zschack, P., Sprung, M., Narayanan, S. \& Sandy, A. R. Direct measurement of antiferromagnetic domain fluctuations. Nature 447, 68-71 (2007).

40 Im, M.-Y., Fischer, P., Kim, D.-H., Lee, K.-D., Lee, S.-H. \& Shin, S.-C. Direct real-space observation of stochastic behavior in domain nucleation process on a nanoscale. Adv. Mater. 20, 1750-1754 (2008).

41 Aron, C., Barci, D. G., Cugliandolo, L. F., Arenas, Z. G. \& Lozano, G. S. Magnetization dynamics: path-integral formalism for the stochastic Landau-Lifshitz-Gilbert equation. J. Stat. Mech. 2014, 09008 (2014).
42 Kakazei, G. N., Ilyn, M., Chubykalo-Fesenko, O., Gonzalez, J., Serga, A. A., Chumak, A. V., Beck, P. A., Laege, B., Hillebrands, B. \& Guslienko, K. Y. Slow magnetization dynamics and energy barriers near vortex state nucleation in circular permalloy dots. Appl. Phys. Lett. 99, 052512 (2011).

$43 \mathrm{Im}$, M.-Y., Lee, K.-S., Vogel, A., Hong, J.-I., Meier, G. \& Fischer, P. Stochastic formation of magnetic vortex structures in asymmetric disks triggered by chaotic dynamics. Nat. Commun. 5, 5620 (2014).

(c) (i) This work is licensed under a Creative Commons Attribution 4.0 International License. The images or other third party material in this article are included in the article's Creative Commons license, unless indicated otherwise in the credit line; if the material is not included under the Creative Commons license, users will need to obtain permission from the license holder to reproduce the material. To view a copy of this license, visit http:// creativecommons.org/licenses/by/4.0/

(C) The Author(s) 2017 